\title{
An Indirect Mixed-Sensitivity Approach To Microgravity Vibration Isolation: \\ The Exploitation of Kinematic Coupling In Frequency-Weighting Design-Filter Selections
}

\author{
R. David Hampton \& Mark S. Whorton
}

\section{Abstract:}

Many space-science experiments need an active isolation system to provide them with the requisite microgravity environment. The isolation systems planned for use with the International Space Station have been appropriately modeled using relative position, relative velocity, and acceleration states. In theory. frequency-weighting design filters can be applied to these state-space models, in order to develop optimal $\mathrm{H}_{2}$ or mixed-norm controllers with desired stability and performance characteristics. In practice, however, the kinematic coupling among the various states can lead, through the associated frequency-weighting-filters, to conflicting demands on the Riccati design "machinery." The results can be numerically ill-conditioned regulator and estimator Riccati equations and/or reduced intuition in the design process. In addition, kinematic coupling can result in a redundancy in the demands imposed by the frequency weights. Failure properly to account for this type of coupling can lead to an unnecessary increase in controller dimensionality and, in turn, controller complexity. This paper suggests a rational approach to the assignment of frequencyweighting design filters, in the presence of the kinematic coupling among states that exists in the microgravity vibration isolation problem.

\section{Introduction}

The international space community has devoted a great deal of attention, in recent years, to the difficult problem of providing a microgravity environment for space-science experiments. Measurements on space platforms have made it clear that even the sub-milli-g acceleration levels ("g-jitter") present on orbit can severely contaminate experimental results $[1,2,3,4]$. Passive isolation systems alone are inadequate [5]. They are not effective below about one $\mathrm{Hz}$; nor can they isolate against direct disturbances to an experimental payload. Rattlespace constraints pose a further limitation $[6,7]$, since the orbits of orbiter and payload typically differ, at some points, by several feet.

Various active isolation systems exist or are under development to address the microgravity vibration isolation problem $[8,9,10,11]$. Relative-position and absolute-acceleration measurements are typically available for control of these systems, for all of which linearized analytical system models are available [e.g., 12]. The control outputs are typically the currents to voice-coil- (Lorentz-force-) actuated electromagnetic or electromechanical actuators. It is in the use of these state-space models for optimal controller design that the problem addressed in this paper arises.

\section{Controller-design problem}

During the typical optimal-controller design process the designer must impose frequency-weighting design filters on the system model to shape the closed-loop sensitivity and complementary sensitivity functions. Upon choosing these filters he often finds that seemingly innocuous choices produce unintended, and typically undesirable, effects. Some choices inexplicably result in ill-conditioned Riccati equations. Others escape this difficulty, only to produce results seemingly unrelated to the design-filter choices. The design process may devolve into a semi-, non-, or even counter-intuitive labor of trial and error.

One source of these effects is kinematic coupling among states, such as that between relative position and absolute acceleration. For example, in a single-degree-of-freedom (SDOF) microgravity vibration-isolation problem, a frequency-weighting filter on relative-position state $x-d$ ( $x$ : experiment position, $d$ : orbiter, or experiment-rack position) corresponds inherently to an implicit weighting on absolute-acceleration state $\ddot{x}$, since any weighing on $x-d$ weights both $x$ and any of its time-derivatives. The presence of rack displacement $d$ in the relative-position state further (and quite substantially) clouds the effect of the kinematic coupling. This state coupling can cause a corresponding coupling among the associated state frequency-weightings or, alternatively, among the sensitivity- and complementary-sensitivity frequency-weightings, depending on the design approach in use. The results can be numerically ill-conditioned Riccati equations and/or reduced 
design-process intuition. The problem is that the kinematic coupling can lead, through these design filters, to conflicting or redundant demands on the Riccati design "machinery." Failure properly to account for this coupling can lead to greater-than-needed controller size and complexity.

\section{Research objectives}

The goal of the present research was to provide sufficient insight into the design-filter selection process for a multiple-input-multiple-output (MIMO) microgravity vibration-isolation problem, to permit positive control (steering) of the loop-shaping process. The investigation was narrowed to the case of $H^{2}$ synthesis, and the results were tested on a SDOF system.

The research traversed the following steps. (1) The interrelationships among frequency-weighting design filters were examined, on a quadratic performance index, for a typical microgravity vibration-isolation problem. (2) The performance index was re-expressed in terms of appropriately related (i.e., kinematically uncoupled) sensitivity- and complementary-sensitivity-function frequency-weighting filters. (3) The relationship was determined between the state weighting filters and the sensitivity- and complementary-sensitivity-function weighting filters. (4) These results were evaluated for aiding in state weighting filter selection. (5) The insights were tested on a simple SDOF microgravity vibration-isolation problem, to evaluate their utility in facilitating the design process.

\section{Microgravity vibration-isolation problem}

\subsection{System Equations}

Consider a typical microgravity vibration isolation system, described in state-space form, with relativeposition, relative velocity, and absolute acceleration states. For example, the SDOF system shown in Figure 1 contains all the salient features. [Multiple-degree-of-freedom (MDOF) systems, such as MIM and g-LIMIT, require additional translational, and corresponding rotational, states and disturbances; but the equations of motion (EOMs) and the cost functionals have analogous forms.] From Figure 1 the EOM for the system is

$$
f_{1}-k(x-d)-c(\dot{x}-\dot{d})-\alpha u=m \ddot{x},
$$

where $f_{1}$ is the direct disturbance force; $m$ is the flotor (isolated experiment) mass; $k$ and $c$ are umbilical stiffness and damping, respectively; $\alpha$ is the Lorentz-force constant of proportionality between control current and actuator Lorentz force; and $d$ and $x$ are, respectively, the rack and experiment displacements from their equilibrium (relaxed-umbilical) positions.

Define the following states: relative position: $z_{1}=x-d$,

$$
\text { relative velocity: } z_{2}=\dot{x}-\dot{d} \text {, }
$$

and (bandpass-filtered)

$$
\text { absolute acceleration: } Z_{3}(s)=\left(\frac{\omega_{h}}{s+\omega_{h}}\right) s^{2} X(s) \text {. }
$$

Then the EOMs can be written in standard state-space form $\underline{\dot{z}}=A \underline{z}+B u+E \underline{f}$,

$$
\text { where } \quad \underline{z}=\left[\begin{array}{lll}
z_{1} & z_{2} & z_{3}
\end{array}\right]^{T} \text { and } \underline{f}=\left[\begin{array}{ll}
\ddot{d} & f_{1} / m
\end{array}\right]^{T} \text {. }
$$

\subsection{Kinematically Coupled Frequency-Weighting Filters \& Performance Index}

Define frequency-weighted state and control vectors as follows:

$$
\underline{Z}_{f}(s)=W_{Z}(s) \underline{Z}(s) \text { and } \underline{U}_{f}(s)=W_{U}(s) \underline{U}(s) \text {. }
$$

Then controller design by $H^{2}$ synthesis (or, alternatively, as a subproblem of a mixed-norm design approach) uses a frequency-weighted quadratic performance index that has the following forms in the

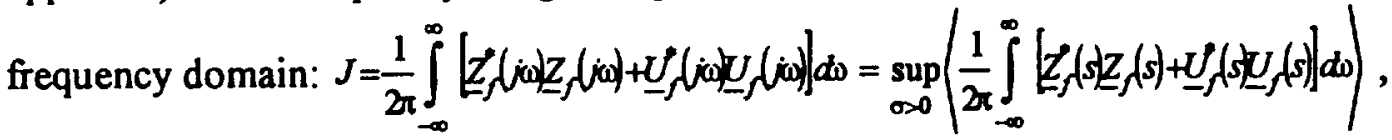

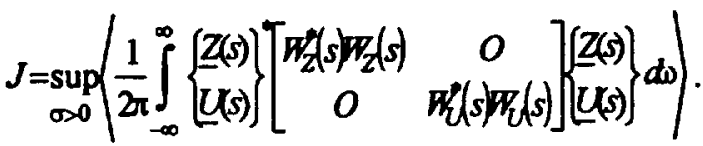


In particular, $\quad \underline{Z}_{f}(s)=\left\{\begin{array}{l}Z_{f 1}(s) \\ Z_{f 2}(s) \\ Z_{f 3}(s)\end{array}\right\}:=\left[\begin{array}{ccc}W_{1}(s) & 0 & 0 \\ O & W_{2}(s) & 0 \\ O & O & W_{3}(s)\end{array}\right]\left\{\begin{array}{l}Z_{1}(s) \\ Z_{2}(s) \\ Z_{3}(s)\end{array}\right\}=\left\{\begin{array}{l}W_{1}(s) Z_{1}(s) \\ W_{2}(s) Z_{2}(s) \\ W_{3}(s) Z_{3}(s)\end{array}\right\}$

where $Z_{1}(s)=X(s)-D(s)$

is the relative position vector,

$Z_{2}(s)=s[X(s)-D(s)]=s Z_{1}(s)$ is the relative velocity vector,

$Z_{3}(s)=\frac{\omega_{h} s^{2} X(s)}{s+\omega_{h}} \approx s^{2} X(s) \quad$ represents the absolute acceleration for sufficiently large $\omega_{h}$,

and the state frequency-weighting design filters are $W_{i}(s)(i=1,2,3)$.

Notice in particular that $Z_{2}(s)=s Z_{1}(s)$, and that $Z_{3}(s) \approx s^{2} Z_{1}(s)+s^{2} D(s)$. This means that although, in principle, one could shape the closed-loop system by judicious choices of the frequency-weighting design filters, in practice the kinematic coupling among the states clouds the filter choices. They cannot be chosen independently, without the possibility of imposing conflicting or redundant demands on the Riccati design machinery. Conflicting demands can lead to ill-conditioning; redundant demands, to an unnecessary increase in controller dimensionality. Either can lead to a loss in design intuition.

\subsection{Kinematically Decoupled Frequency-Weighting Filters, for Cheap Control}

This problem can be addressed as follows. Rewrite the cost-functional as

$$
J=\sup _{\infty \rightarrow 0}\left(\frac{1}{2 \pi} \int_{-\infty}^{\infty}\left[I_{Z}(s)+I_{U}(s)\right] d \omega\right)
$$

where the state energy term is

$$
\begin{aligned}
I_{Z} & =\underline{Z}_{f}^{*}(s) \underline{Z}_{f}(s) ; \\
I_{U} & =\underline{U}_{f}^{*}(s) \underline{U}_{f}(s) .
\end{aligned}
$$

and the control energy term,

Consider the integrand for the case of "cheap control," to limit the focus to the cost of the frequency-weighted state energy term alone. The frequency-weighted state energy term is

$$
\begin{aligned}
& \left.I_{Z}=\underline{Z}_{f}^{*} \underline{Z}_{f}=(X-D)^{*} W_{1}^{*} W_{1}+\left(s W_{2}\right)^{*}\left(s W_{2}\right)\right](X-D)+\left(s^{2} X\right)^{*}\left(W_{3}^{*} W_{3}\right)\left(s^{2} X\right), \\
& \text { or } \quad I_{Z}=\left[s^{2}(X-D)^{*}\left[\left(\frac{W_{1}}{s^{2}}\right)^{*}\left(\frac{W_{1}}{s^{2}}\right)+\left(\frac{W_{2}}{s}\right)^{*}\left(\frac{W_{2}}{s}\right)\right]\left[s^{2}(X-D)\right]+\left(s^{2} X\right)^{*}\left(W_{3}^{*} W_{3}\right)\left(s^{2} X\right) .\right.
\end{aligned}
$$

Let $I$ represent an identity matrix of appropriate dimensions. If system input sensitivity- and complementary sensitivity functions are defined, respectively, by $X(s)=T_{X D} D(s)$ and $S_{X D}=I-T_{X D}$, the frequency-weighted state-energy term can be expressed as follows:

$$
I_{Z}=\left(s^{2} D\right) *\left(s_{X D}^{*} V_{s}^{*} V_{S} s_{X D}+T_{X D}^{*} V_{T}^{*} V_{T} T_{X D}\right)\left(s^{2} D\right)
$$

where $\quad V_{s}^{*} V_{s}=\left(\frac{W_{1}}{s^{2}}\right)^{*}\left(\frac{W_{1}}{s^{2}}\right)+\left(\frac{W_{2}}{s}\right)^{*}\left(\frac{W_{2}}{s}\right) \quad$ and $\quad V_{T}^{*} V_{T}=W_{3}^{*} W_{3}$.

\subsection{Relationships Between State Filters and Sensitivity Filters}

$$
\text { Observe that } \quad V_{S}=\left[\left(\frac{W_{1}}{s^{2}}\right)^{*}\left(\frac{W_{1}}{s^{2}}\right)+\left(\frac{W_{2}}{s}\right)^{*}\left(\frac{W_{2}}{s}\right)\right]^{1 / 2} \text { and } V_{I}=W_{3}
$$

are weightings on sensitivity function $S_{X D}$ and complementary sensitivity function $T_{X D}$, respectively. Equivalently, $V_{S}$ and $V_{T}$ can be viewed as respective weightings on $S_{s^{2} X, s^{2} D}$ and $T_{s^{2} X, s^{2} D}$, relating rack and experiment accelerations. By properly considering Equations $(26,29$, and 30$)$, the designer can now effectively address the kinematic coupling among states in his choices of state frequency-weighting design filters for loop-shaping. In particular, if he chooses his filters by considering the effects on $S_{s^{2} X, s^{2} D}\left(S_{X D}\right)$ of $W_{1} / s^{2}$ and/or $W_{2} / s$, rather than the direct effects of $W_{1}$ and $W_{2}$ on the performance index, the filter choices will become apparent. $W_{3}$ is chosen based on its effect on $T_{s^{2} X, \Omega^{2} D}\left(T_{X D}\right)$; its effect on $S_{s^{2} X, \Omega^{2} D}\left(S_{X D}\right)$ is only 
indirect, through Equation (25), and can be neglected, provided the separation in frequency between the demands of $V_{S}$ and $V_{T}$ is sufficent.

\subsection{Design Filter Considerations for a MicrogravityIsolation Problem}

The control engineer seeks to shape the closed-loop acceleration transmissibility so as to pass lowfrequency acceleration disturbances (to accommodate rattlespace constraints), to reject intermediate-range acceleration disturbances, to dampen resonances, and to "turn off" the controller below frequencies of unmodeled system dynamics. These requirements translate into (1) unit transmissibility to indirect acceleration disturbances (i.e., unity $T_{s^{2} x, s^{2} D}$ ) for low frequencies, say, below a comer frequency of about 0.01 $\mathrm{Hz}$, (2) rapid rolloff of transmissibility above the corner frequency, for good attenuation up to about $10 \mathrm{~Hz}$, and (3) controller turm-off (low controller gains) above, say, $100 \mathrm{~Hz}$.

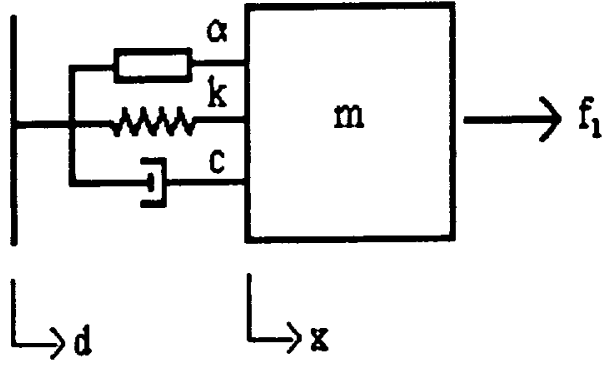

Figure 1. A SDOF Microgravity Isolator

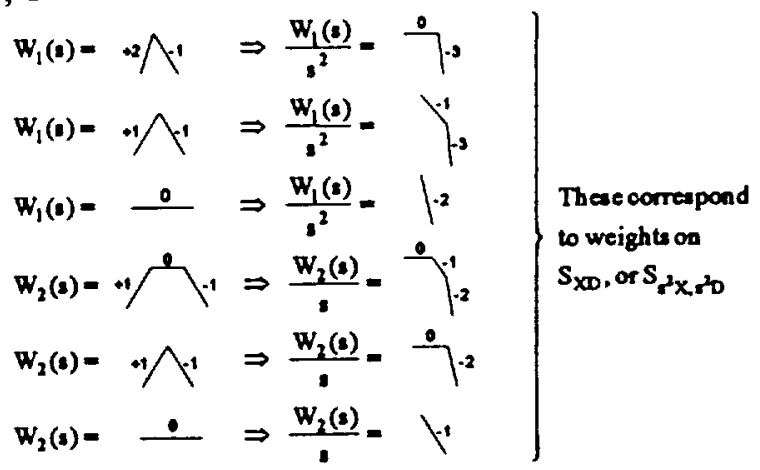

Table 1. Reasonable Weighting-Function Candidates

\section{Reasonable Filter Choices}

Table 1 presents some reasonable possible choices for $W_{1}$ (or $W_{2}$ ), based on the resulting effective filters $W_{1} / s^{2}\left(W_{2} / s\right)$. The latter (effective) filters correspond to frequency weights $V_{s}$ on $S_{s^{2} \times, x^{2} D}$. Notice that integrating or band-pass types of filter shapes are logical candidates for $V_{s}$, since these will call for good

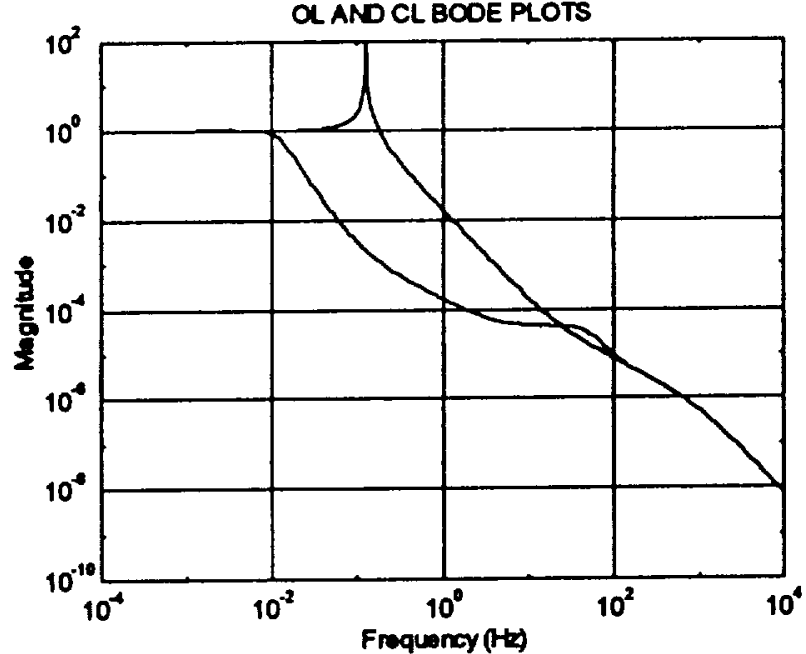

Figure 2. Transmissibility to Rack Accelerations

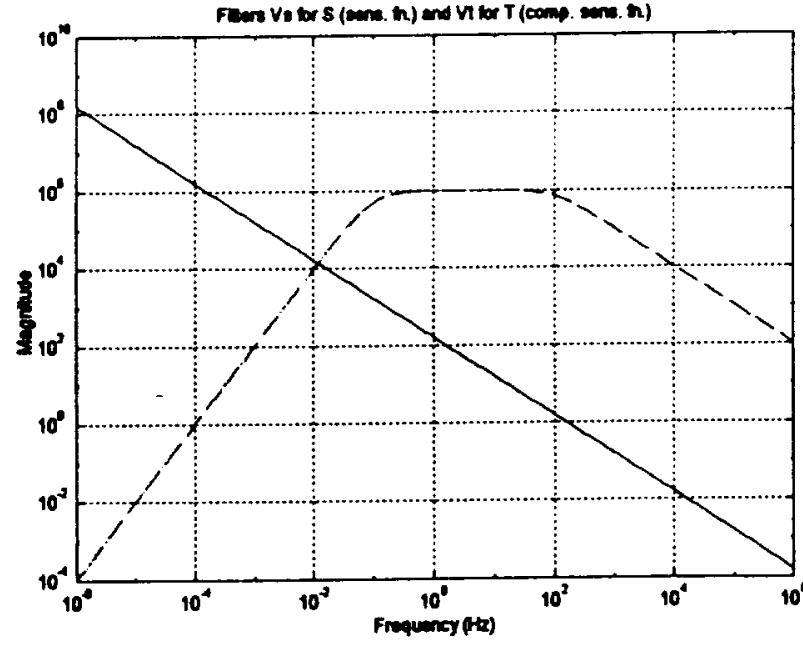

Figure 3. Filters $V_{S}$ and $V_{T}$ acceleration tracking at low frequencies without conflicting with the call of $V_{T}$ for good disturbanceattenuation at higher frequencies. Logical choices for $W_{3}\left(V_{T}\right)$ are bandpass filters, with higher initial slopes calling for steeper initial rolloffs in transmissibility $\left(T_{s^{2} X, s^{2} D}\right)$ above the comer frequency. Filter $W_{3}\left(V_{T}\right)$ should roll off at higher frequencies to allow the controller to tum off. In practice, controller turn-off can be determined by the rejoining of transmissibility plots for the open-loop and closed-loop systems. 


\section{A SDOF Test Case}

Filters selected from Table 1 were applied to a SDOF test case (see Fig. 1) having the following parameter values: $m=75 \mathrm{lbm}, k=1.5 \mathrm{lbf} / \mathrm{ft}, c=0.01 \mathrm{lbf} \cdot \mathrm{s} / \mathrm{ft}, \alpha=2 \mathrm{lbf} / \mathrm{amp}$. Typical open- and closed-loop plots of the complementary sensitivity function $\left(T_{s^{2} X, s^{2} D}\right)$ are shown above in Figure 2, for which the frequency-weighting filters were $W_{1}:$ zero; $W_{2}$ : constant; $W_{3}:$ band-pass, with sequential legs having slopes of $+2,0$, and -1 ; and $W_{U}$ : stair-step, with sequential legs having slopes of $0,+2$, and 0 . Resulting effective filters on $S_{s^{2} X, s^{2} D}$ and $T_{s^{2} X, s^{2} D}$ are shown in Figure 3. Observations from the test case are as follows:

(1) The location of the comer frequency for the complementary sensitivity plot $T_{s^{2} X . s^{2} D}$ can be varied by varying the relative weights on $S_{s^{2} X, s^{2} D}$ and $T_{s^{2} X, s^{2} D}$ due to $V_{S}$ and $V_{T}$, respectively. Higher relative weighting on $S_{s^{2} X, s^{2} D}$ tends to shift the comer frequency to the right.

(2) The rate of the rolloff (slopc) of $T_{s^{2} X, s^{2} D}$ is determined by the rate of ascent (slope) of the $1^{x}$ leg of the weighting filter $W_{3}$ on $T_{s^{2} X, s^{2} D}$ : they typically are the same, with a change in sign.

(3) The controller bandwidth is reduced by rolling off the weighting (penalty) on $S_{s^{2} X, s^{2} D}$ and $T_{s^{2} X, s^{2} D}$, and by ramping up (while still maintaining the requisite zero final slope) the penalty $W_{U}$ on control current $u$.

(4) Numerical difficulties in solving the regulator and estimator Riccati equations were essentially nonexistent; numerical ill-conditioning occurred rarely with rationally chosen (see section 5 above) frequencyweighting filter choices, and then only when very large factors (several orders of magnitude) were used to multiply those filters.

\section{Suggested Design Approach}

A suggested design approach is described below:

(1) Choose the sensitivity-function frequency-weighting filter shape(s) for good nominal performance at low frequencies, and at DC. By "good nominal performance" for the microgravity vibration isolation problem, one means unit transmissibility, for the nominal plant, to indirect (umbilical-transmitted) acceleration disturbances below a corner frequency driven by rattlespace constraints.

(2) Choose the complementary-sensitivity frequency-weighting filter shape(s) for good nominal performance in intermediate and higher frequencies. By "good nominal performance" in these regions, for the microgravity isolation problem, one means rapid roll-off (again, for indirect acceleration disturbances) and low acceleration transmissibilities above the corner frequency, for the nominal plant. This corresponds also to designing for good stability robustness.

(3) Choose frequency-weighting filters to force the controller to "tum off" (i.e., to add negligible energy into the closed-loop system) above frequencies of interest. This is accomplished (a) by choosing state-(or corresponding sensitivity- and complementary-sensitivity function) design-filter weightings that place minimal demands for control action at higher frequencies, and (b) by choosing controller design-filter weightings that exact heavy penalties on control at higher frequencies.

\section{Acknowledgements}

The authors wish to thank NASA Marshall Space Flight Center for partial funding of this work, through the NASA ASEE Summer Faculty Fellowship Program.

\section{References}

1. Nelson, Emily S., "An Examination of Anticipated g-Jitter on Space Station and Its Effects on Materials Processes," NASA TM-103775, April 1991.

2. DelBasso, S., "The International Space Station Microgravity Environment," AIAA-96-0402, January 1996.

3. "System Specification for the International Space Station," Specification Number SSP41000, Rev. D, Nov. 1, 1995, NASA Johnson Space Center. 
4. DeLombard, R, Bushnell, G. S., Edberg, D., Karchmer, A. M., and Tryggvason, B. V., "Microgravity Environment Countermeasures Panel Discussion," AlAA-97-0351, January 1997.

5. Grodsinsky, Carlos M. and Brown, Gerald V., "Nonintrusive Inertial Vibration Isolation Technology for Microgravity Space Experiments," NASA TM-102386, ALAA-90-0741, January 1990.

6. Knospe, C. and Allaire, P. "Limitations on Vibration Isolation for Microgravity Space Experiments," Journal of Spacecraft and Rockets, Vol. 27, No. 6, Nov.-Dec. 1990, pp. 642-646.

7. Knospe, C. and Allaire, P., "Limits on the Isolation of Stochastic Vibration for Microgravity Space Experiments," Journal of Spacecraft and Rockets, Vol. 28, No. 2, March-April 1991, pp. 229-237.

8. Edberg, D., Goucher, R., Schenck, D., Nurre, G., Whorton, M., Kim, Y., and Alhorn, D., "Results of the STABLE Microgravity Vibration Isolation Flight Experiment," Guidance and Control, Vol. 92, 1996, pp. 567-581.

9. Tryggvason, B., Stewart, W., de Carufel, J., and Vezina, L., "Acceleration Levels and Operation of the Microgravity Vibration Isolation Mount (MIM) on the Shuttle and the Mir Space Station," AIAA-99-0578, January 1999.

10. Bushnell, G., "STS-79 Final Report, RME-1313/ARIS," Boeing Document No. SK683-61855-1, December 1996.

11. Whorton, M. S., "g-LIMTT: A Vibration Isolation System for the Microgravity Science Glovebox," AIAA99-0577, January 1999.

12. Hampton, R. D., Tryggrason, B. V., DeCarufel, J., Townsend, M. A., Wagar, W. O., "The Microgravity Vibration Isolation Mount: A Dynamic Model for Optimal Controller Design," Proceedings of the MAG '97 Industrial Conference and Exhibition, August 1997. 